\title{
Fabrication of Metal-Gated Carbon Nanotube Emitter Arrays Assisted by Focused Ion Beam and Chemical Vapor Deposition
}

\author{
Jianfeng Wu, Lifeng Dong, Jeremy Petty, Chiaching Pan and Jun Jiao \\ Physics Department, Portland State University, Portland, OR 97207, U.S.A.
}

Carbon nanotubes (CNTs) have excellent electronic and structural properties. The high aspect ratio and small tip radius of CNTs make them especially suitable as potential electron sources [1]. The objective of this study is to develop a technique for fabricating triode-type CNT field emitter arrays, where an integrated extraction gate is built between the cathode and the anode. The gate electrode is placed close to the cathode, making the gate to emitter distance small. As a result, the gate can attract or repel electrons with modest changes in gate voltage (positive or negative), enabling greater control over the emission current [2].

Our novel approach for fabricating metal-gated carbon nanotube emitters is based on our previous work of using combined techniques of focused ion beam (FIB) and chemical vapor deposition to fabricate CNTs on designed locations [3]. In this study, we initially fabricated Si posts within arrays of Si holes on a piece of Si wafer by FIB. Then, we used the ion-induced decomposition of ferrocene to deposit $\mathrm{Fe}$ catalyst on the top of each Si post. The processed substrate was placed into a CVD reactor. SEM results showed that CNTs grew from the top of every Si post [Fig. 1]. Some straight carbon nanotubes grew from the Si posts as shown in Fig. 1-3. These promising results led us to develop new procedures as follows to improve this technique for fabricating metal-gated carbon nanotube emitter arrays (MGCNTEAs).

Instead of using Si wafers, we used a sandwich structure containing a single layer, $1 \mu \mathrm{m}$ thick, of silicon dioxide $\left(\mathrm{SiO}_{2}\right)$ on a silicon substrate. A conductive adhesion layer of TiN, $50 \mathrm{~nm}$ thick, was deposited on the top of the $\mathrm{SiO}_{2}$ by sputtering, and this was followed by a $120 \mathrm{~nm}$ thick Pt layer which was designed to act as the gate electrode. Arrays of holes were generated by the FIB, and ion induced decomposition of ferrocene was performed to deposit Fe catalyst into these holes. Fig. 4 shows one of these substrate arrays. Elemental composition of our prepared sample in Fig. 4 prior to CNT growth was done using energy dispersive X-ray spectrometry (EDS). The spectrum shown in Fig. 5 indicates the presence of $\mathrm{Si}, \mathrm{Ti}, \mathrm{Pt}$ and $\mathrm{O}$, all materials used in our substrate preparation process. The whole substrate was then put into a CVD reactor for CNT growth. An FEI Sirion field emission SEM was then used to characterize the samples. Fig. 3 demonstrates the formation of CNTs inside of the holes as well as from the hole-walls. Sidewall growth will affect the electrical properties of the arrays. In order to avoid this type of growth, we used the FIB to remove the catalyst deposits from the hole-walls. Fig. 6 shows that CNT growth occurred only from Fe catalyst in the bottom of holes after the FIB cleaning process.

The morphologies of metal-gated carbon nanotube emitters could be affected by the dimension of the holes, the size of the catalyst particles, and CVD parameters. Further study is underway to optimize the fabrication process and to investigate the electrical properties of MGCNTEAs [4].

References

[1] Wei, L. et al, J. Vac. Sci. Technol. B 18 (2000) 2704.

[2] Hsu, David S.Y. and Jonathan Shaw, Appl. Phys. Lett. 80 (2002) 118.

[3] J. Jiao, L. F. Dong, S. Foxley, C. L. Mosher et al, Microsc. Microana. 9 (2003) 516.

[4] Financial support for this research was provided in part by the NSF under awards No. ECS0455937, ECS-0217061 and DMR-0353738 (REU Site). 

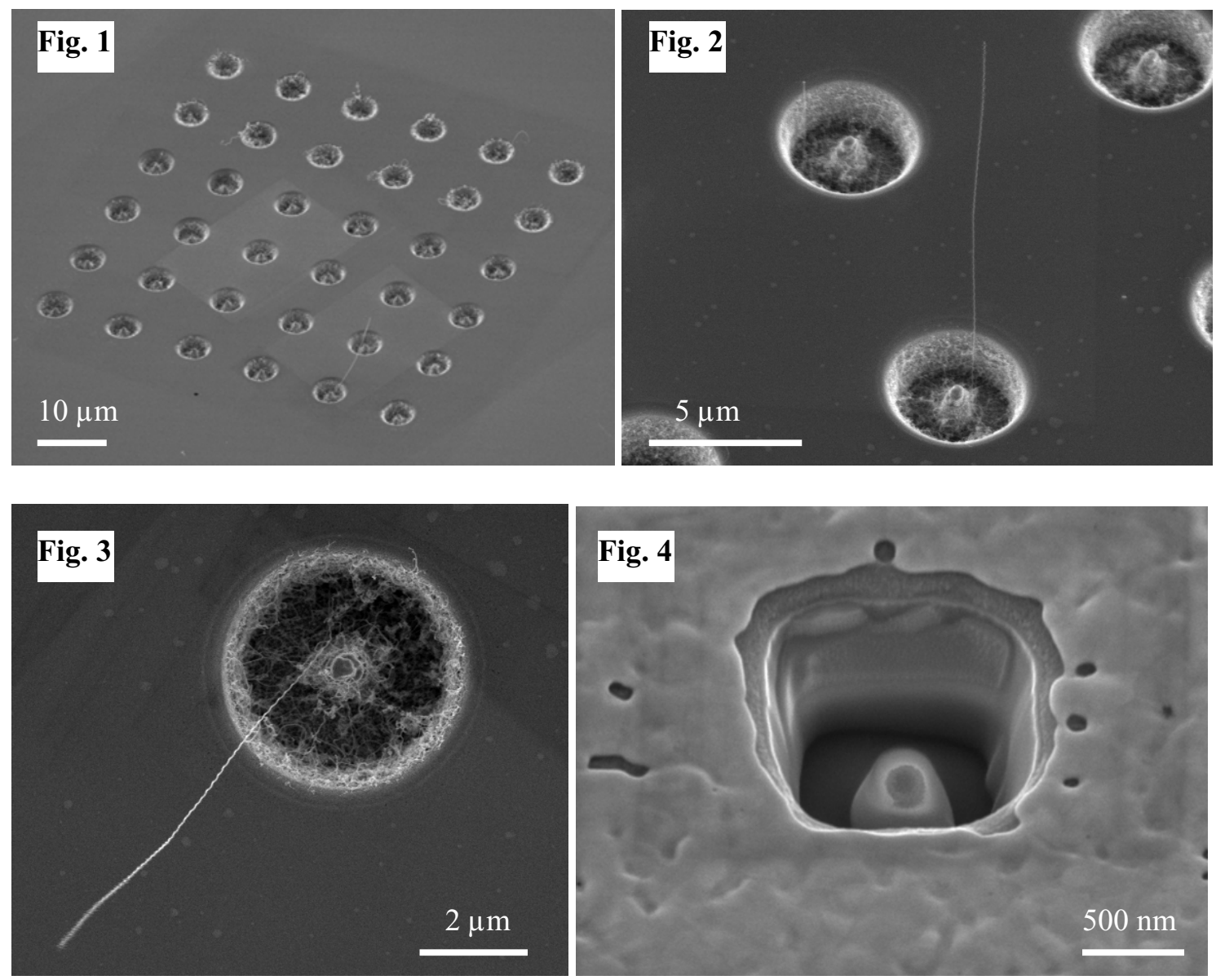

Fig. 5
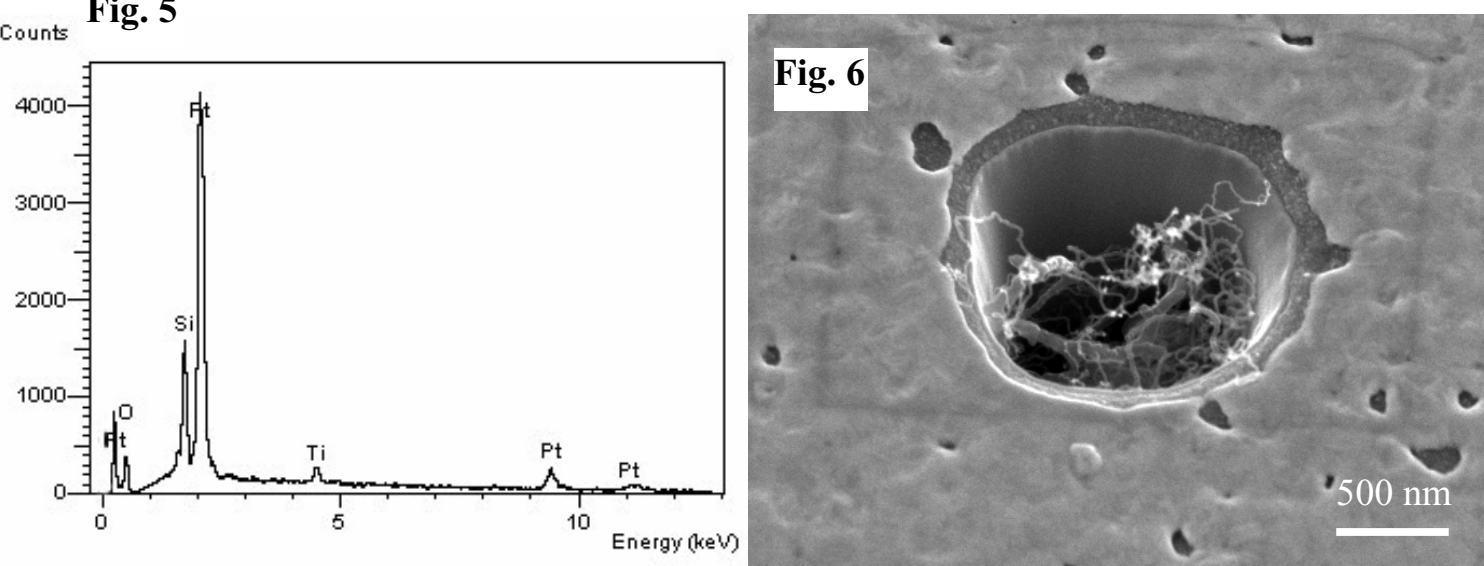

Fig.1 CNTs grew from the top of every Si post within arrays of Si holes on a piece of Si wafer.

Fig.2 A straight carbon nanotube grew from the Si posts.

Fig.3 The formation of CNTs inside of the holes as well as from the hole-walls.

Fig.4 One site from an array shows $\mathrm{Fe}$ deposition from ion-induced decomposition of ferrocene.

Fig.5 EDS spectrum indicates the presence of $\mathrm{Si}, \mathrm{Ti}, \mathrm{Pt}$ and $\mathrm{O}$, all materials used in our substrate preparation process.

Fig.6 CNT growth occurred only from Fe catalyst in the bottom of holes after the FIB cleaning process. 